\title{
The role of TGF- $\beta$ pathway alterations in immune regulation as a potential pan-cancer biomarker in immunotherapy
}

\author{
Yan Liang ${ }^{1 \#}$, Wei Li ${ }^{1 \#}$, Bing Qian ${ }^{2}$ Jie Ming ${ }^{3}$, Zhengyi Zhao ${ }^{4}$, Zhengqing Yan ${ }^{4}$, Xiaochen Zhao ${ }^{4}$ \\ Shiqing Chen ${ }^{4}$, Yongmei Yin ${ }^{1,5}$
}

${ }^{1}$ Department of Oncology, The First Affiliated Hospital of Nanjing Medical University, Nanjing, China; ${ }^{2}$ Nanjing Medical University Affiliated Cancer Hospital Jiangsu Cancer Hospital, Jiangsu Cancer Institute, Nanjing, China; ${ }^{3}$ The Affiliated Hospital of Qingdao University, Qingdao, China; ${ }^{4}$ The Medical Department, 3D Medicines Inc., Shanghai, China; ${ }^{5}$ Jiangsu Key Lab of Cancer Biomarkers, Prevention and Treatment, Collaborative Innovation Center for Cancer Medicine, Nanjing Medical University, Nanjing, China

Contributions: (I) Conception and design: Y Yin, Y Liang, W Li; (II) Administrative support: Y Yin; (III) Provision of study materials or patients: Y Yin, Y Liang, W Li, B Qian, J Ming; (IV) Collection and assembly of data: Y Liang, W Li, B Qian, J Ming; (V) Data analysis and interpretation: Z Zhao, Z Yan, X Zhao, S Chen; (VI) Manuscript writing: All authors; (VII) Final approval of manuscript: All authors.

\#These authors contributed equally to this work and should be considered co-first authors.

Correspondence to: Yongmei Yin, MD. Department of Oncology, The First Affiliated Hospital of Nanjing Medical University, No. 300 Guangzhou Road, Nanjing 210029, China; Jiangsu Key Lab of Cancer Biomarkers, Prevention and Treatment, Collaborative Innovation Center for Cancer Medicine, Nanjing Medical University, Nanjing, China. Email: ym.yin@hotmail.com.

Background: Depending on the context, the transforming growth factor beta (TGF- $\beta$ ) signaling pathway is involved in opposing cell processes of tumor suppression and tumor promotion. However, the effects of TGF- $\beta$ pathway on immunotherapy efficacy have not yet been systematically investigated.

Methods: In this study, we have extracted the available data of whole-exome sequencing, messenger RNA (mRNA) expression, baseline characterization, and prognosis information of 10,912 pan-cancer patients from The Cancer Genome Atlas to explore the role of TGF- $\beta$ pathway in immune regulation. Formalin-fixed, paraffin-embedded tissue samples from 6,717 Chinese cancer patients assayed by next-generation sequencing (NGS) were used as a validation cohort (3DMed cohort). Data sets from the public MSK (Memorial Sloan Kettering Cancer Center) cohort $(\mathrm{N}=1,610)$ were used to explore the association of TGF- $\beta$ pathway with immunotherapy effects.

Results: The results showed that TGF- $\beta$ pathway alteration was significantly correlated with high microsatellite instability (MSI), high tumor mutational burden, and high neoantigen burden (TNB) $(\mathrm{P}<0.001$ for each). Consistently, the pathway mutations were associated with distinct patterns of immune-related gene expression and tumor-infiltrating immune cells. Patients with TGF- $\beta$ pathway mutations exhibited significantly worse prognosis than did the wild-type patients regardless of the interventions [overall survival (OS): hazard ratio (HR) 1.20; 95\% confidence interval (CI): 1.08-1.33; P=0.001]. However, when treated with immune checkpoint inhibitors (ICIs), superior survival benefit was observed in patients from the mutation group versus the wild-type group (OS: HR 0.73; 95\% CI: 0.61-0.88; P=0.001).

Conclusions: Collectively, our study suggested that mutations in TGF- $\beta$ pathway may be associated with positive immune regulation and better efficacy of immunotherapy.

Keywords: Next-generation sequencing (NGS); solid tumors; immune regulation; microsatellite instability (MSI)

Submitted Sep 03, 2021. Accepted for publication Nov 09, 2021.

doi: $10.21037 / \mathrm{atm}-21-5138$

View this article at: https://dx.doi.org/10.21037/atm-21-5138

\footnotetext{
$\wedge$ ORCID: 0000-0002-7544-9916.
} 


\section{Introduction}

Immune checkpoint blockades (ICBs) have already demonstrated the promising clinical activity in many solid tumors. However, just a small part of cancer patients could benefit from the ICB treatment. Several molecular biomarkers may help to select patients who more likely derive benefit from ICBs, such as PD-L1 expression (1-3), mismatch repair-deficient (dMMR)/microsatellite instability-high (MSI-H) $(4,5)$ and tumor mutational burden (TMB) (6). Especially, dMMR/MSI-H and TMB have been FDA-approved pan-cancer biomarkers for ICB treatment in advanced solid tumors. Recently, more and more biomarkers have been explored to predict ICB efficacy, especially mutations in different pathways, such as DNA damage response (DDR) pathway (7), NOTCH signaling (8), and PBAF complex (9), but these biomarkers have limited utility.

The seemingly split personality of transforming growth factor beta (TGF- $\beta$ ) pathway in cancer development has been widely reported in recent past years. TGF- $\beta$ pathway is considered to switch from tumor suppressive in the early stages of carcinogenesis to metastasis and tumor promoting as the tumor progresses $(10,11)$. Treatment strategies that block TGF- $\beta$ signaling in advanced-stage or metastatic cancers are currently being evaluated in clinical trials (12), such as several novel TGF- $\beta$ signaling inhibitors. Another interesting avenue of research involves investigating the role of the TGF- $\beta$ superfamily in the immune microenvironment and its relevance to cancer. TGF- $\beta$ can regulate both innate and adaptive immunity via multiple mechanisms $(13,14)$. Aberrant upregulation of the pathway has been shown to correlate with tumor metastasis and cancer progression through the potential induction of epithelial-mesenchymal transition (EMT) from either canonical or noncanonical TGF- $\beta$ downstream signaling in late-stage tumors $(15,16)$. In this regard, an increasing number of ongoing and upcoming studies will be exploring the combined strategies of anti-TGF- $\beta$ and immune checkpoint blockades (ICBs) in cancer treatment.

TGF- $\beta$ signaling is associated with broad immune evasion through the inhibition of $\mathrm{T}$ cell immunity and other immune functions, leading to immune tolerance in various tumors, such as (17), metastatic urothelial cancer (mUC) (18), non-small cell lung cancer (NSCLC) (16), hepatocellular carcinoma (HCC) (19), melanoma and bladder carcinoma (20). For example, through the analysis of RNA sequencing data of 298 pretreatment tumor samples from a large phase 2 trial (IMvigor210) of mUC, a signature of TGF- $\beta$ signaling was found to attenuate atezolizumab response by contributing to the exclusion of T cells (18). Combined administration of anti-TGF- $\beta$ and anti-programmed death-ligand 1 (PD-L1) was observed to provoke vigorous antitumor immunity and tumor regression. Another phase 2 study from Feun et al. showed a high baseline TGF- $\beta$ level to be significantly correlated with poor progression-free survival $(\mathrm{PFS})(\mathrm{P}=0.008)$ and overall survival $(\mathrm{OS})(\mathrm{P}=0.005)$ outcomes after pembrolizumab in 28 evaluable patients with advanced HCC (19). Similarly, Chakravarthy et al. (20) reported that TGF- $\beta$ activation was linked to C-ECM (cancer-associated ECM) dysregulation, which was shown to be a predictor of the failure of programmed cell death protein 1 (PD-1) blockade in two cohorts (one melanoma cohort and one bladder cancer cohort). Interestingly, Ankur's study also found TGF- $\beta$ 1/2 expression alone could not predict immunotherapy failure, while a C-ECM-based model that considered TGF- $\beta$ activation together with cancer-associated fibroblasts (CAF abundance) showed independently satisfactory predictive performance of the treatment outcomes with PD-1 blockades (20).

Previous work suggests that most mutations in TGF- $\beta$ pathway activators are gain of function (21). Thus, TGF- $\beta$ pathway gene alterations might be related to a net effect of overall increase in the pathway activity. Notably, the overall role of TGF- $\beta$ pathway alteration in pan-cancer immunotherapy effects remains unclear, but this is an important issue to clarify for the development of treatment strategies in tumors with aberrant TGF- $\beta$ signaling. The present study was aimed to investigate the overall effect TGF- $\beta$ pathway alteration in immune regulation and its predictive performance in immunotherapy in a pan-cancer setting. We present the following article in accordance with the REMARK reporting checklist (available at https:// dx.doi.org/10.21037/atm-21-5138).

\section{Methods}

\section{Patient coborts}

Data sets from 3 independent pan-cancer cohorts with clinical and genomic data were used in this study. Available data of whole-exome sequencing, messenger RNA (mRNA) expression, baseline characterization, and prognosis information of a total of 10,912 patients with 31 tumor types were acquired from The Cancer Genome 
Table 1 List of the number of patients with different tumor types in the 3DMed and immune cohorts

\begin{tabular}{|c|c|}
\hline Tumor type & $\mathrm{N}$ \\
\hline \multicolumn{2}{|l|}{ 3DMed cohort } \\
\hline Bladder urothelial carcinoma & 172 \\
\hline Breast invasive carcinoma & 328 \\
\hline $\begin{array}{l}\text { Cervical squamous cell carcinoma and } \\
\text { endocervical adenocarcinoma }\end{array}$ & 166 \\
\hline Cholangiocarcinoma & 369 \\
\hline Colon adenocarcinoma & 612 \\
\hline Esophageal carcinoma & 138 \\
\hline Kidney renal clear cell carcinoma & 110 \\
\hline Liver hepatocellular carcinoma & 314 \\
\hline Lung adenocarcinoma & 1,694 \\
\hline Lung squamous cell carcinoma & 402 \\
\hline Others & 571 \\
\hline Ovarian serous cystadenocarcinoma & 237 \\
\hline Pancreatic adenocarcinoma & 467 \\
\hline Prostate adenocarcinoma & 86 \\
\hline Rectum adenocarcinoma & 349 \\
\hline Stomach adenocarcinoma & 609 \\
\hline Uterine corpus endometrial carcinoma & 93 \\
\hline Total & 6,717 \\
\hline \multicolumn{2}{|l|}{ Immune cohort } \\
\hline Bladder cancer & 211 \\
\hline Breast cancer & 41 \\
\hline Cancer of unknown primary & 85 \\
\hline Colorectal cancer & 109 \\
\hline Esophagogastric cancer & 118 \\
\hline Glioma & 116 \\
\hline Head and neck cancer & 129 \\
\hline Melanoma & 313 \\
\hline Non-small cell lung cancer & 344 \\
\hline Renal cell carcinoma & 143 \\
\hline Skin cancer, non-melanoma & 1 \\
\hline Total & 1,610 \\
\hline
\end{tabular}

Atlas (TCGA) (http://www.cbioportal.org/) for genomic characterization. The abundance of the tumor immune infiltration of the samples estimated from RNA sequencing data was obtained from the public data set released on the web-accessible resource Tumor Immune Estimation Resource (TIMER) (http://cistrome.org/TIMER). Data of microsatellite instability (MSI) for TCGA samples were obtained from the public data set in a previous publication based on 5,930 cancer exomes from 18 cancer types at more than 200,000 microsatellite loci (22).

The 3DMed (3D Medicines Inc.) cohort with 6,717 formalin-fixed paraffin-embedded (FFPE) tumor specimens with matched blood control of Chinese cancer patients with more than 17 tumor types were enrolled in this study (Table 1). The samples were collected and underwent nextgeneration sequencing (NGS) for the identification of genomic mutations and MSI status from January 01, 2017, to July 23, 2019. Baseline characteristics of the patients were collected. There were $56.2 \%$ male patients and $43.8 \%$ female patients in the overall population. The median age for the cohort was 59 [interquartile range (IQR), 50-66].

Genomic data, baseline characterization, and OS data of 1,662 advanced cancer patients treated with immune checkpoint inhibitors (ICIs) whose tumors underwent NGS using a 468-gene targeted panel (MSK-IMPACT ${ }^{\mathrm{TM}}$, MSKintegrated mutation profiling of actionable cancer targets) were adopted from the previously published data set (23) (immune cohort, Table 1).

All procedures performed in this study involving human participants were in accordance with the Declaration of Helsinki (as revised in 2013). The study was approved by the institutional review board of The First Affiliated Hospital of Nanjing Medical University (No. 2018-MD334) and informed consent was taken from all the patients.

\section{$N G S$}

The pathological diagnosis of the specimens was confirmed by hematoxylin and eosin (HE) staining. To be considered as a qualified sample, the specimen needed to be $\geq 1 \mathrm{~mm}$ and the percentage of tumor cells needed to be over $20 \%$. Subsequently, 50-200 ng of DNA extracted from the samples were broken into 200 bp fragments and then subjected to NGS platform Illumina (San Diego, CA, USA) 
Nextseq 500 to $>500 \times$ coverage (24).

Tumor mutation burden (TMB) was defined as somatic mutation counts in coding region per megabase of genome examined. Genes including TGFBR2, ACVR1B, ACVR $2 A$, INHBA, SMAD2, SMAD3, SMAD4, IGF2, RUNX1, $S T A T 3, T E R T$, and $V E G F A$ have been reported to be the core members in the TGF- $\beta$ pathway network and to act as cancer-related genes (21); they were thus selected to be included for analysis. A TGF- $\beta$ pathway mutation was defined as the presence of at least one pathway gene with identified mutational types (missense mutation or truncating mutation), including nonsense mutation, nonstop mutation, frameshift insertion, frameshift deletion, and splice site mutation.

\section{Statistical analysis}

Survival analysis was performed using Kaplan-Meier curves, and the $\mathrm{P}$ value was determined with the log-rank test. The hazard ratio (HR) was determined through Cox regression. All reported $\mathrm{P}$ values were 2 -tailed, and a $\mathrm{P}$ value less than 0.05 was considered significant.

\section{Results}

\section{Landscape of TGF- $\beta$ patbway mutation across multiple tumors}

A total of 10,912 patients with 31 tumor types in TCGA cohort and 6,717 patients with 17 tumor types in 3DMed cohort were analyzed (Table 1). Of the overall population, there were $32.6 \%$ in $3 \mathrm{DMed}$ cohort and $14.8 \%$ in TCGA cohort that harbored at least 1 mutation of TGF- $\beta$ pathway genes. TGF- $\beta$ pathway mutations were more commonly found in the digestive system tumors in both cohorts. The highest prevalence of the pathway mutations was present in colon adenocarcinoma (COAD) (38.3\%), stomach adenocarcinoma (STAD) $(33.6 \%)$, pancreatic adenocarcinoma (PAAD) (31.5\%), rectum adenocarcinoma (READ) (30.9\%), and uterine corpus endometrial carcinoma (UCEC) (29.4\%) in TCGA cohort; and COAD (77.0\%), bladder urothelial carcinoma (BLCA) $(47.7 \%)$, STAD (47.6\%), cholangiocarcinoma (CHOL) $(43.1 \%)$, UCEC (43.0\%), PAAD (38.3\%), and READ (36.7\%) in the $3 \mathrm{DMed}$ cohort. The most common mutated gene in the pathway was $S M A D 4$ with a frequency of 0.075 , followed by TGFBR2 (0.039), ACVR2A (0.031), and CDH1 (0.030) in the 3DMed cohort (Figure 1A).

\section{Association between TGF- $\beta$ pathway mutations and cancer genomic features}

TGF- $\beta$ pathway mutations exhibited a significant correlation with a high MSI across multiple tumors in the TCGA $(\mathrm{P}<0.001)$ and 3DMed $(\mathrm{P}<0.001$, Figure $1 B)$ cohorts. Notably, the significance was consistently observed in most individual tumor types, and nonsignificant correlations were observed in the others partially due to their limited sample sizes (Figure 1B).

Similarly, patients with altered TGF- $\beta$ pathway showed significantly higher levels of tumor TMB in both the TCGA $(\mathrm{P}<0.001$; Figure $2 A)$ and $3 \mathrm{DMed}(\mathrm{P}<0.001$; Figure $2 B)$ cohorts, which was consistently observed across tumor types. It is widely acknowledged that tumors with a high TMB level are able to express more antigens that contribute to immunogenicity, and thus we further conducted the analysis with the neoantigen data sets from TCGA. As expected, significantly higher levels of neoantigen burden (TNB) were observed in patients with altered TGF- $\beta$ pathway $(\mathrm{P}<0.001$; Figure $2 C)$. Notably, these positive correlations between TGF- $\beta$ pathway with either high TMB or high TNB were consistently observed in patients with a microsatellite stable (MSS) status $(\mathrm{P}<0.001$; Figure $2 D)$, and the possibility that such correlations were contributed by the MSI status was excluded.

Next, we investigated the association between TGF- $\beta$ pathway single-nucleotide variants (SNV) mutations with an immune-related gene expression pattern. Based on the analysis, the pathway mutations were associated with significantly higher expression levels of a broad repertoire of immune-related genes, most of which are immune stimulatory genes. Meanwhile, pathway-altered patients exhibited significantly lower expression levels of EDNRB and VEGFB, which were both reported to be involved in immune inhibition (Figure $3 A$ ), indicating a net immunepromoting effect of TGF- $\beta$ pathway alterations.

To further evaluate the tumor-immune interactions, data of the tumor-infiltrating immune cells for samples in TCGA cohort were adopted for analysis. Higher levels of CD8 $(\mathrm{P}<0.001)$, dendritic cells (DC) $(\mathrm{P}<0.001)$, neutrophils $(\mathrm{NEU})(\mathrm{P}<0.001)$, and significantly lower levels of $\mathrm{B}$ cells $(\mathrm{P}<0.001)$ were found in the pathway-mutated samples versus the wild-type ones (Figure $3 B$ ), indicating a network of positive immune regulatory inputs. 


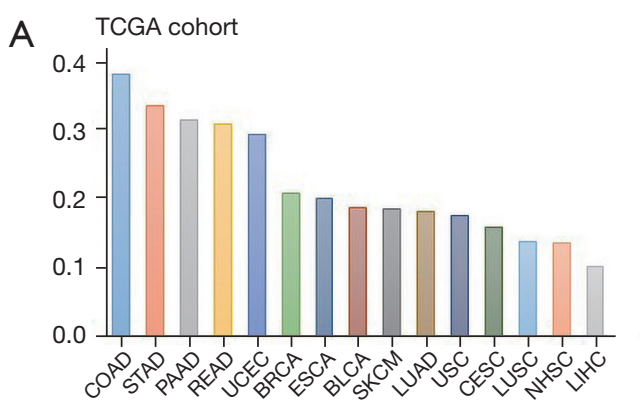

B

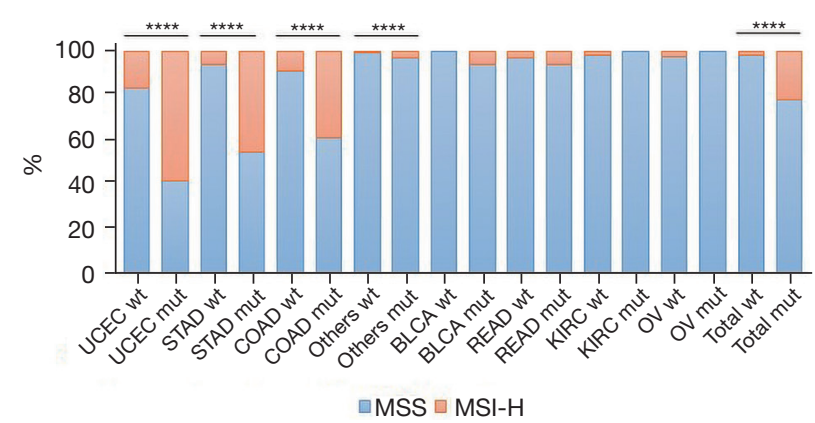

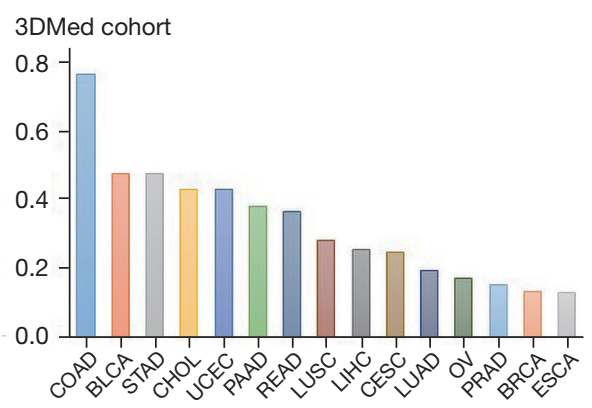

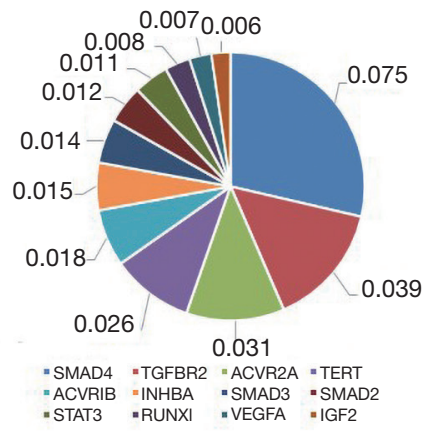

3DMed cohort

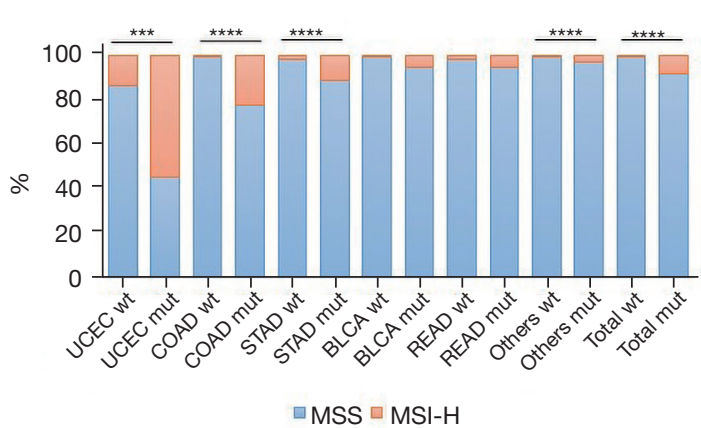

Figure 1 Distribution of TGF- $\beta$ pathway alterations in cancer patients and its association with MSI status. (A) Landscape of TGF- $\beta$ pathway alterations in pan-cancer cohorts of TCGA (left panel) and 3DMed (middle panel), and the prevalence of mutated genes in the pathway in 3DMed cohort (right panel). (B) The association of TGF- $\beta$ pathway alterations with MSI status across various solid tumors in TCGA cohort (left panel) and 3DMed cohort (right panel). Only the tumor types with an overall MSI level of no less than $1 \%$ as derived from the TCGA data set are shown for both cohorts; the other tumor types were combined into the others for presentation. ${ }^{* *}, \mathrm{P}<0.001$; ${ }^{* * *}, \mathrm{P}<0.0001$. TGF- $\beta$, transforming growth factor beta; MSI, microsatellite instability; TCGA, The Cancer Genome Atlas.

\section{TGF- $\beta$ pathway mutations associated with immunotherapy for cancer treatment}

We further investigated the role of TGF- $\beta$ pathway in cancer with the survival data from patients in a pancancer setting. As expected, patients with TGF- $\beta$ pathway mutations exhibited significantly worse prognosis than did the wild-type patients [median OS 89.3 vs. 96.9 months; HR $1.20 ; 95 \%$ confidence interval (CI): 1.08-1.33; $\mathrm{P}=0.001$; Figure $3 C$ ] regardless of the interventions in TCGA cohort. However, when treated with ICIS, superior survival benefit was observed in patients in the mutation group versus the wild-type group (median OS $32.0 \mathrm{vs}$. 17.0 months; HR 0.73; 95\% CI: 0.61-0.88; $\mathrm{P}=0.001$; Figure $3 D$ ) in the immune cohort, indicating that cancer patients harboring TGF- $\beta$ pathway mutations may benefit more from immunotherapy.

\section{Discussion}

We systematically explored the role of aberrant TGF- $\beta$ pathway in immune features and their immunotherapy effects. TGF- $\beta$ pathway alteration was significantly correlated with high MSI, high TMB, high TNB, and distinct patterns of immune-related gene expression and tumor-infiltrating immune cells. These results suggest the potential predictive value of TGF- $\beta$ pathway alterations in improved immunotherapy outcomes in patients with various solid tumors, which may provide clues for the future development of clinical therapeutic strategies. More importantly, these alterations, as a negative prognostic factor, exhibited a positive predictive role for immunotherapy in pan-cancer patients, which provides novel insights into the future development of TGF- $\beta$ pathway-related treatment strategies. 

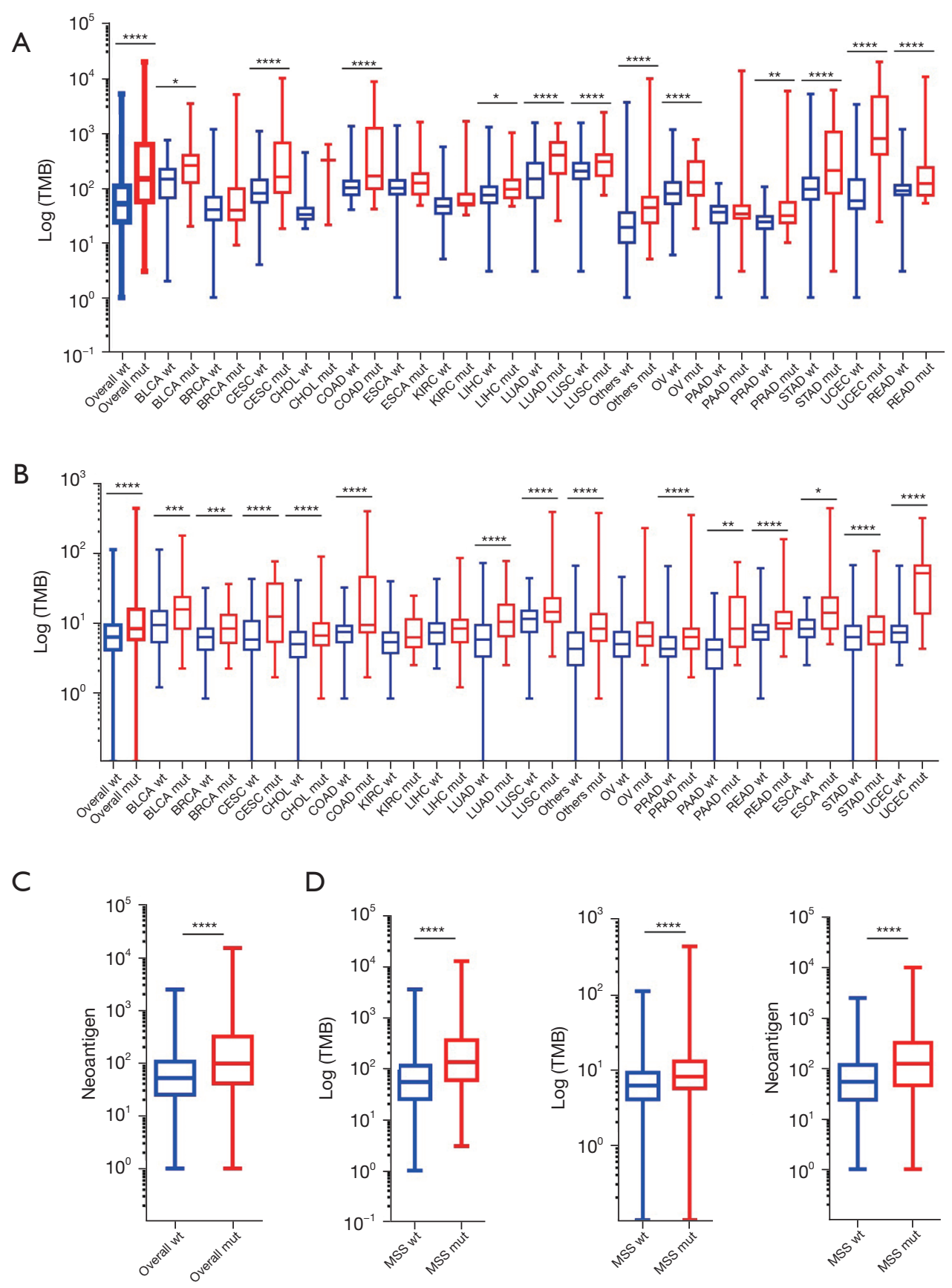

Figure 2 Association of TGF- $\beta$ pathway alterations with TMB and TNB. (A,B) Association of TGF- $\beta$ pathway alterations with TMB in TCGA cohort (A) and the 3 DMed cohort (B) across different tumor types. (C) Association of TGF- $\beta$ pathway alterations with TNB in TCGA cohort. (D) The association of TGF- $\beta$ pathway alterations with TMB in TCGA cohort (left panel) and the 3DMed cohort (middle panel), and its association with TNB in TCGA cohort (right panel) for patients with a MSS status. *, $\mathrm{P}<0.05 ;{ }^{* *}, \mathrm{P}<0.01$; ${ }^{* * *}, \mathrm{P}<0.001$; ${ }^{* * *}, \mathrm{P}<0.0001$. TGF- $\beta$, transforming growth factor beta; TMB, tumor mutational burden; TNB, neoantigen burden; TCGA, The Cancer Genome Atlas; MSS, microsatellite stable. 

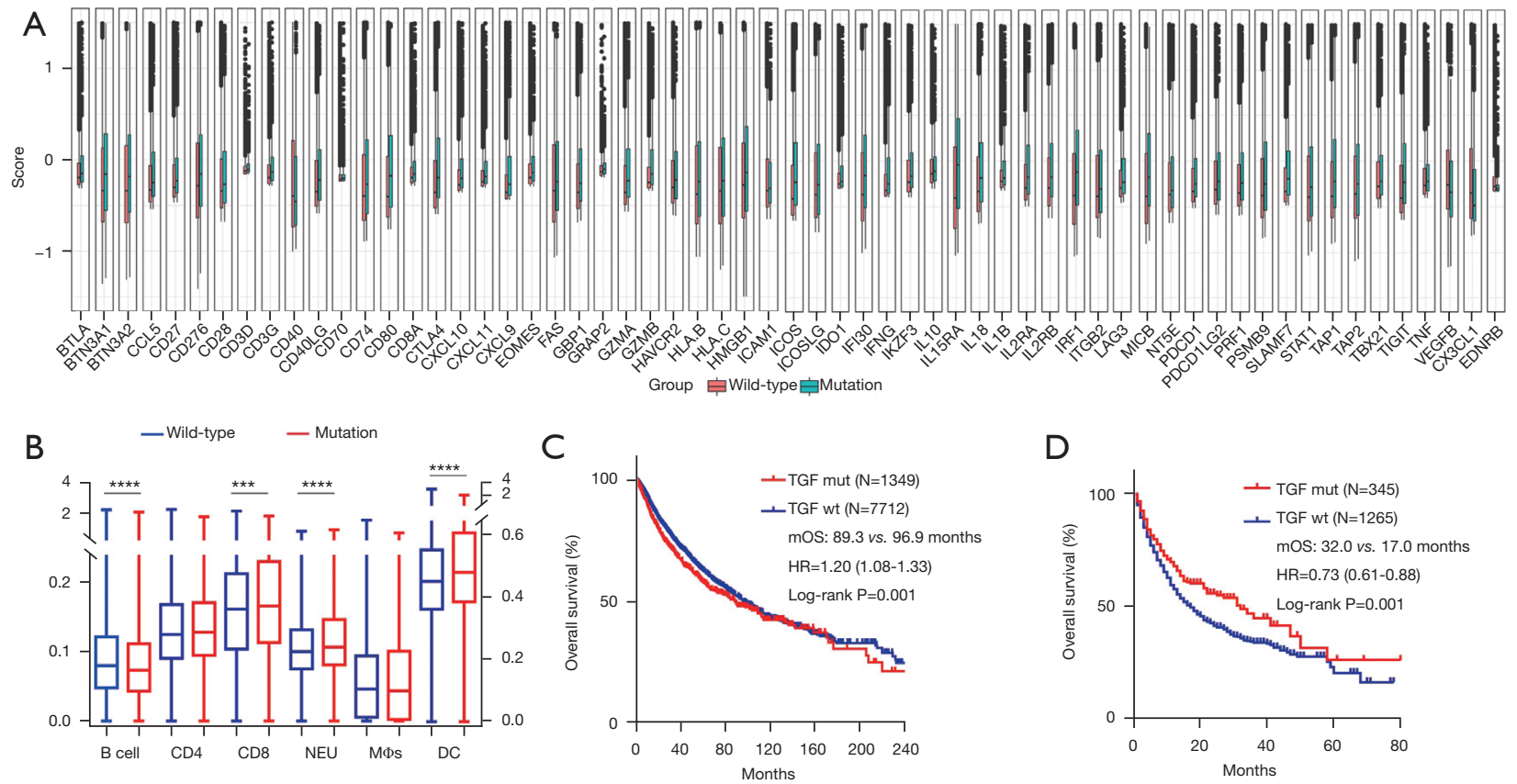

Figure 3 The effect of TGF- $\beta$ pathway alterations in immune profiles and immunotherapy treatment outcomes. (A) Regulation of the immune-related gene expression patterns upon the altered TGF- $\beta$ pathway in TCGA cohort. (B) Association of TGF- $\beta$ pathway alterations and tumor-infiltrating cells in TCGA cohort. (C) Prognosis value of TGF- $\beta$ pathway alterations in overall survival for pan-cancer patients in TCGA cohort. (D) The predictive role of TGF- $\beta$ pathway alterations in overall survival for cancer patients treated with immune checkpoint inhibitors in the immune cohort. ${ }^{* * *}$, $\mathrm{P}<0.001 ;{ }^{* * * *}, \mathrm{P}<0.0001$. TGF- $\beta$, transforming growth factor beta; TCGA, The Cancer Genome Atlas.

TGF- $\beta$ suppresses adaptive and innate immune responses by regulating many immune cell types (10). TGF- $\beta$ promotes the expansion of Treg cells, and inhibits the generation and function of effector $T$ cells and antigenpresenting dendritic cells. In addition, TGF- $\beta$ inhibits NK cells and regulates macrophages and neutrophils. TGF- $\beta$ pathway gene alterations have been found in approximately $10-50 \%$ of solid cancers $(10,21,25)$, which accounts for a nonnegligible amount of target patients. Our analysis demonstrated that TGF- $\beta$ pathway alterations were commonly located in various solid tumors, and the pathway was most frequently aberrant in digestive system tumors, which is consistent with previous reports (21). Other research has suggested that TGF- $\beta$ signaling is a potent inducer of growth inhibition in benign epithelia, premalignant tumors, and some early cancerous tissues, and it has been used for patients with advanced stages of cancer progression and immune suppression $(10,20,26)$. Studies have suggested that TGF- $\beta$ pathway could impair MHC class II expression, thus potentially impairing priming of CD4 T cells, and suppresses the activity of cytotoxic lymphocytes by inhibiting the differentiation, proliferation, and effector functions of CD8T cells and natural killer (NK) cells (27). In this work, patients with TGF- $\beta$ pathway mutations exhibited significantly worse prognosis than did the wild-type patients. Unexpectedly, pathway alteration was significantly associated with a favorable immune profile, including significantly higher levels of CD8 $(\mathrm{P}<0.001)$, DCs and NEUs, as well as significantly lower levels of B cells in pathway-mutated samples versus the wild-type ones.

The relationship between TGF- $\beta$ pathway and ICBs have been previously explored. The present study represents the first of its kind to examine the role of TGF- $\beta$ pathway gene alterations in immunotherapy in patients with various solid tumors. In view of the retrospective nature of the study, some potential bias might exist. First, different cohort types should be evaluated in future research. Second, only SNVs were considered in this study, and further investigations of the other variant types in the pathway genes are needed. Third, more validation cohorts in solid tumors are required 
to verify the predictive value of pathway alteration in immunotherapy treatment outcomes.

\section{Conclusions}

Collectively, our study demonstrated that TGF- $\beta$ pathway alteration was significantly correlated with high MSI, high $\mathrm{TMB}$, high TNB, and distinct patterns of immune-related gene expression and tumor-infiltrating immune cells. These results support the potential predictive value of TGF- $\beta$ pathway alterations in improved immunotherapy outcomes in patients with various solid tumors, which may provide insight into the future development of clinical therapeutic strategies.

\section{Acknowledgments}

Funding: This study was funded by the National Natural Science Foundation of China (No. 81972484 to YY), the Collaborative Innovation Center for Tumor Individualization Focuses on Open Topics (No. JX21817902/008 to YY), the High-Level Innovation Team of Nanjing Medical University (No. JX102GSP201727 to YY), the Project of China Key Research and Development Program Precision Medicine Research (No. 2016YFC0905901 to YY), the Key Medical Talents (No. ZDRCA2016023 to YY), the National Key Research and Development Program of China (No. ZDZX2017ZL-01 to YY), and the Wu Jieping Foundation (No. 320.6750.17006 to $\mathrm{YY})$.

\section{Footnote}

Reporting Checklist: The authors have completed the REMARK reporting checklist. Available at https://dx.doi. org/10.21037/atm-21-5138

Data Sharing Statement: Available at https://dx.doi. org/10.21037/atm-21-5138

Conflicts of Interest: All authors have completed the ICMJE uniform disclosure form (available at https://dx.doi. org/10.21037/atm-21-5138). ZZ, ZY, XZ, and SC are from 3D Medicines Inc. The other authors have no conflicts of interest to declare.

Ethical Statement: The authors are accountable for all aspects of the work in ensuring that questions related to the accuracy or integrity of any part of the work are appropriately investigated and resolved. All procedures performed in this study involving human participants were in accordance with the Declaration of Helsinki (as revised in 2013). This study was approved by the Ethics Committee of The First Affiliated Hospital of Nanjing Medical University (No. 2018-MD-334), and all patients provided written informed consent.

Open Access Statement: This is an Open Access article distributed in accordance with the Creative Commons Attribution-NonCommercial-NoDerivs 4.0 International License (CC BY-NC-ND 4.0), which permits the noncommercial replication and distribution of the article with the strict proviso that no changes or edits are made and the original work is properly cited (including links to both the formal publication through the relevant DOI and the license). See: https://creativecommons.org/licenses/by-nc-nd/4.0/.

\section{References}

1. Reck M, Rodríguez-Abreu D, Robinson AG, et al. Pembrolizumab versus Chemotherapy for PD-L1Positive Non-Small-Cell Lung Cancer. N Engl J Med 2016;375:1823-33.

2. Hu Z, Lu L, Fei Z, Lv D. Association between clinicopathological features and prognosis significance of PD-L1 expression in small cell lung cancer patients: a systemic review and meta-analysis. Transl Cancer Res 2020;9:5508-16.

3. Yi M, Jiao D, Xu H, et al. Biomarkers for predicting efficacy of PD-1/PD-L1 inhibitors. Mol Cancer 2018;17:129.

4. Overman MJ, McDermott R, Leach JL, et al. Nivolumab in patients with metastatic DNA mismatch repair-deficient or microsatellite instability-high colorectal cancer (CheckMate 142): an open-label, multicentre, phase 2 study. Lancet Oncol 2017;18:1182-91.

5. Marabelle A, Le DT, Ascierto PA, et al. Efficacy of Pembrolizumab in Patients With Noncolorectal High Microsatellite Instability/Mismatch Repair-Deficient Cancer: Results From the Phase II KEYNOTE-158 Study. J Clin Oncol 2020;38:1-10.

6. Marabelle A, Fakih M, Lopez J, et al. Association of tumour mutational burden with outcomes in patients with advanced solid tumours treated with pembrolizumab: prospective biomarker analysis of the multicohort, openlabel, phase 2 KEYNOTE-158 study. Lancet Oncol 
2020;21:1353-65.

7. Wang Z, Zhao J, Wang G, et al. Comutations in DNA Damage Response Pathways Serve as Potential Biomarkers for Immune Checkpoint Blockade. Cancer Res 2018;78:6486-96.

8. Zhang K, Hong X, Song Z, et al. Identification of Deleterious NOTCH Mutation as Novel Predictor to Efficacious Immunotherapy in NSCLC. Clin Cancer Res 2020;26:3649-61.

9. Hakimi AA, Attalla K, DiNatale RG, et al. A pancancer analysis of PBAF complex mutations and their association with immunotherapy response. Nat Commun 2020;11:4168.

10. Batlle E, Massagué J. Transforming Growth Factor- $\beta$ Signaling in Immunity and Cancer. Immunity 2019;50:924-40.

11. Bierie B, Moses HL. Tumour microenvironment: TGFbeta: the molecular Jekyll and Hyde of cancer. Nat Rev Cancer 2006;6:506-20.

12. Derynck R, Turley SJ, Akhurst RJ. TGF $\beta$ biology in cancer progression and immunotherapy. Nat Rev Clin Oncol 2021;18:9-34.

13. Kelly A, Houston SA, Sherwood E, et al. Regulation of Innate and Adaptive Immunity by TGF $\beta$. Adv Immunol 2017;134:137-233.

14. Sanjabi S, Oh SA, Li MO. Regulation of the Immune Response by TGF- $\beta$ : From Conception to Autoimmunity and Infection. Cold Spring Harb Perspect Biol 2017;9:a022236.

15. Deckers M, van Dinther M, Buijs J, et al. The tumor suppressor Smad4 is required for transforming growth factor beta-induced epithelial to mesenchymal transition and bone metastasis of breast cancer cells. Cancer Res 2006;66:2202-9.

16. Eser PÖ, Jänne PA. TGF $\beta$ pathway inhibition in the treatment of non-small cell lung cancer. Pharmacol Ther 2018;184:112-30.

17. Metelli A, Wu BX, Fugle CW, et al. Surface Expression of TGF $\beta$ Docking Receptor GARP Promotes Oncogenesis and Immune Tolerance in Breast Cancer. Cancer Res

Cite this article as: Liang Y, Li W, Qian B, Ming J, Zhao Z, Yan Z, Zhao X, Chen S, Yin Y. The role of TGF- $\beta$ pathway alterations in immune regulation as a potential pan-cancer biomarker in immunotherapy. Ann Transl Med 2021;9(22):1660. doi: 10.21037/atm-21-5138
2016;76:7106-17.

18. Mariathasan S, Turley SJ, Nickles D, et al. TGF $\beta$ attenuates tumour response to PD-L1 blockade by contributing to exclusion of T cells. Nature 2018;554:544-8.

19. Feun LG, Li YY, Wu C, et al. Phase 2 study of pembrolizumab and circulating biomarkers to predict anticancer response in advanced, unresectable hepatocellular carcinoma. Cancer 2019;125:3603-14.

20. Chakravarthy A, Khan L, Bensler NP, et al. TGF- $\beta$ associated extracellular matrix genes link cancer-associated fibroblasts to immune evasion and immunotherapy failure. Nat Commun 2018;9:4692.

21. Korkut A, Zaidi S, Kanchi RS, et al. A Pan-Cancer Analysis Reveals High-Frequency Genetic Alterations in Mediators of Signaling by the TGF- $\beta$ Superfamily. Cell Syst 2018;7:422-437.e7.

22. Hause RJ, Pritchard CC, Shendure J, et al. Classification and characterization of microsatellite instability across 18 cancer types. Nat Med 2016;22:1342-50.

23. Samstein RM, Lee CH, Shoushtari AN, et al. Tumor mutational load predicts survival after immunotherapy across multiple cancer types. Nat Genet 2019;51:202-6.

24. Su D, Zhang D, Chen K, et al. High performance of targeted next generation sequencing on variance detection in clinical tumor specimens in comparison with current conventional methods. J Exp Clin Cancer Res 2017;36:121.

25. Chen J, Zaidi S, Rao S, et al. Analysis of Genomes and Transcriptomes of Hepatocellular Carcinomas Identifies Mutations and Gene Expression Changes in the Transforming Growth Factor- $\beta$ Pathway. Gastroenterology 2018;154:195-210.

26. Principe DR, Doll JA, Bauer J, et al. TGF- $\beta$ : duality of function between tumor prevention and carcinogenesis. J Natl Cancer Inst 2014;106:djt369.

27. Lazarova M, Steinle A. Impairment of NKG2DMediated Tumor Immunity by TGF- $\beta$. Front Immunol 2019;10:2689.

(English Language Editor: J. Gray) 